\title{
The role of soft quarks in next-to-leading power threshold effects
}

\author{
Melissa van Beekveld* \\ Radboud University Nijmegen, Nikhef \\ E-mail: mcbeekveld@gmail.com
}

Wim Beenakker

Radboud University Nijmegen, University of Amsterdam

Email:w.beenakker@science.ru.nl

\section{Eric Laenen}

University of Amsterdam, Utrecht University, Nikhef

Email:t45@nikhef.nl

\section{Anuradha Misra}

University of Mumbai

Email: anuradha.misra@gmail.com

\section{Chris D. White}

Queen Mary University of London

Email: christopher.whitedqmul.ac.uk

\begin{abstract}
Cross sections in perturbative QCD are plagued by large corrections from soft and collinear radiation. The most singular terms are known to be universal, which allows their resummation to all orders in the coupling. In our work, we have examined the structure of the next-to-singular contributions, which can originate from the emission of both soft quarks and gluons. We show that we can derive a next-to-soft amplitude for both types of emissions. The numerical impact of these contributions on the transverse momentum distribution of the single-photon production process are also discussed.
\end{abstract}

14th International Symposium on Radiative Corrections (RADCOR2019)

9-13 September 2019

Palais des Papes, Avignon, France

\footnotetext{
* Speaker.
} 
Cross sections in perturbative Quantum Chromodynamics (QCD) are plagued by large corrections from soft and collinear radiation. These results from an incomplete cancellation between real and virtual singularities, and thus the appearance of large contributions at all orders in perturbation theory. If $\xi$ denotes a dimensionless kinematic ratio such that $\xi \rightarrow 0$ near threshold, the corresponding differential cross section has the following form

$$
\frac{\mathrm{d} \sigma}{\mathrm{d} \xi} \propto \sum_{n=0}^{\infty}\left(\frac{\alpha_{s}}{\pi}\right)^{n} \sum_{m=0}^{2 n-1}\left[c_{n m}^{R}\left(\frac{\ln ^{m} \xi}{\xi}\right)_{+}+c_{n}^{V} \delta(\xi)+c_{n m}^{\mathrm{NLP}} \ln ^{m} \xi+\ldots\right] .
$$

The first two sets of terms originate from soft and collinear radiation (real or virtual). These are the leading power (LP) contributions in the threshold variable $\xi$ and are localized at $\xi=0$. The contributions have a universal form, which allows for their all-order resummation. The third set of terms in Eq. (1) are the next-to-leading power (NLP) contributions, which are suppressed by a single power of the threshold variable. Although subleading, the increasing precision of both LP resummation and of experimental data makes such terms numerically relevant. Significant progress towards understanding NLP corrections is being made [1, 2, 3, 4, 5, 6, 7, 8, 9, 10, 11, 12, 13, 14, 15, $16,17,18,19,20,21,22,23,24,25,26,27,28,29]$, and a resummation of them at LL in processes where only soft real radiation is present is achieved [30, 31, 32].

In this proceeding contribution, we will report on our progress to determine the origin of NLP terms in an next-to-leading order (NLO) calculation, namely that of prompt photon. We also assess the numerical impact of the NLP terms in the jointly resummed prompt photon production process.

\section{Universal NLO amplitudes for (next-to-)soft radiation}

In Ref. [33], we have shown that the NLP contributions at the first logarithmic order (LL) in an NLO process can be correctly described by

$$
\mathscr{A}^{\mathrm{NLP}}=\mathscr{A}^{\text {gluon }}+\mathscr{A}^{\text {quark }}
$$

The soft gluon and soft quark amplitudes $\left(\mathscr{A}^{\text {gluon }}\right.$ and $\left.\mathscr{A}^{\text {quark }}\right)$ are obtained by making a soft expansion of the matrix element, defined as the expansion where all components of the emitted momentum $k \rightarrow 0$. The (next-to-)soft gluon amplitude then becomes

$$
\begin{aligned}
\mathscr{A}_{\text {gluon }} & =\mathscr{A}_{\text {scal }}+\mathscr{A}_{\text {spin }}+\mathscr{A}_{\text {orb }} \\
& =\sum_{j=1}^{n+2} \frac{g_{s} \mathbf{T}_{j}}{2 p_{j} \cdot k}\left(\mathscr{O}_{\mathrm{scal}, j}^{\sigma}+\mathscr{O}_{\mathrm{spin}, j}^{\sigma}+\mathscr{O}_{\mathrm{orb}, j}^{\sigma}\right) \otimes i \mathscr{M}_{\mathrm{H}}\left(p_{1}, \ldots, p_{i}, \ldots, p_{n+2}\right) \varepsilon_{\sigma}^{*}(k)
\end{aligned}
$$

Here, $\mathscr{M}_{\mathrm{H}}$ denotes the hard scattering matrix element. The first two terms on the right-hand side constitute the scalar and spin contributions respectively. The third term is the orbital angular momentum operator, and $\mathbf{T}_{j}$ a color generator in the appropriate representation. Each of these operators are defined in the Appendix A of Ref. [33]. The strong coupling is denoted by $g_{s}$, and the momentum of the hard line that emits the soft gluon is denoted by $p_{j}$. The scalar contribution starts at LP in the soft expansion, whereas both of the angular momentum contributions start at NLP. The result of Eq. (1.2) has previously been derived [34, 35, 36, 37], and is known as the next-to-soft 

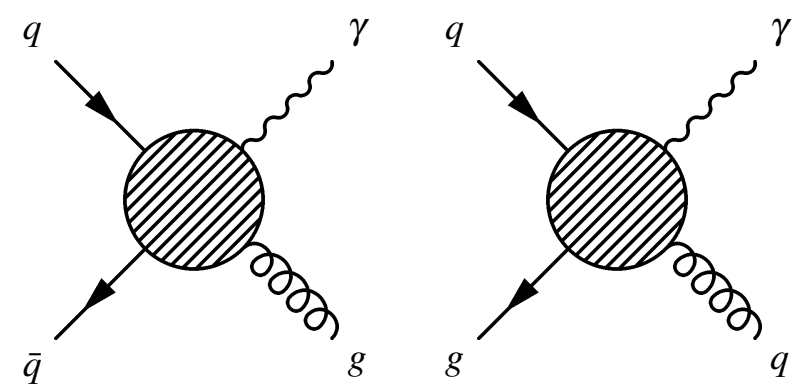

Figure 1: Feynman diagrams for the LO processes $q \bar{q} \rightarrow g \gamma$ (left) and $q g \rightarrow \gamma q$ (right).

theorem.

The second term in Eq. (1.1) is the soft quark amplitude and reads

$$
\mathscr{A}_{\mathrm{NLP}, \mathscr{Q}}=\sum_{j=1}^{n+2} \frac{g_{s}}{2 p_{j} \cdot k} \mathscr{Q}_{j} \otimes i \mathscr{M}_{H, j}\left(p_{1}, p_{2}, \ldots, p_{j}, \ldots, p_{n+2}\right) \text {. }
$$

The operator $\mathscr{Q}_{j}$ is the quark emission operator, which acts on a given external parton line $j$ to produce the emission of a quark or anti-quark. This operator changes the identity of the external line it acts on, therefore we let it act on a collection of different hard scattering matrix elements $\mathscr{M}_{H, j}$, which need to be projected onto the process of interest.

\section{Application of the NLP amplitude to the prompt photon production process}

To test the coverage of the NLP amplitude, we consider the production of a single photon that recoils against a hard parton at NLO, where the latter is unobserved (the prompt photon production process). As this process has 7 partonic subchannels and more than one color structure to consider at NLO, it is an interesting testing ground for our next-to-soft gluon and soft quark formalisms. At leading order (LO), the prompt photon production process $(p p \rightarrow \gamma+X)$ consists of two subprocesses: $q \bar{q} \rightarrow \gamma g$ and $q g \rightarrow q \gamma$, as shown in Fig. 1. In what follows, we will consider the NLO contributions $q \bar{q} \rightarrow \gamma g g$ and $q g \rightarrow \gamma q g$

Considering the $q\left(p_{1}\right) \bar{q}\left(p_{2}\right) \rightarrow \gamma\left(p_{\gamma}\right) g\left(p_{R}\right) g(k)$ process, we find that all NLP contributions up to LL are generated by shifts of the Born squared amplitude $H_{q \bar{q} \rightarrow \gamma g}$

$$
\begin{aligned}
\left\langle\left|\mathscr{A}_{\mathrm{LP}+\mathrm{NLP}, q \bar{q} \rightarrow \gamma g g}\right|^{2}\right\rangle=\frac{Q_{q}^{2} g_{\mathrm{EM}}^{2} g_{s}^{4} C_{F}}{4} C_{A}\left[\left(C_{F}-\frac{1}{2} C_{A}\right)\right. & \frac{2 p_{1} \cdot p_{2}}{\left(p_{1} \cdot k\right)\left(p_{2} \cdot k\right)} \\
& \times H_{q \bar{q} \rightarrow \gamma g}\left(p_{1}+\delta p_{1 ; 2}, p_{2}+\delta p_{2 ; 1}, p_{\gamma}, p_{R}\right)
\end{aligned}
$$

where $g_{\mathrm{EM}}$ is the electromagnetic coupling, and $Q_{q}$ is the charge of the quark that the photon couples to in units of the electron mass. The shifts are defined as

$$
\delta p_{i ; j}^{\alpha} \equiv-\frac{1}{2}\left(k^{\alpha}+\frac{p_{j} \cdot k}{p_{i} \cdot p_{j}} p_{i}^{\alpha}-\frac{p_{i} \cdot k}{p_{i} \cdot p_{j}} p_{j}^{\alpha}\right)
$$


After integrating over the full three-body phase space, we find that the next-to-soft formalism formalism correctly reproduces LL terms at both LP and NLP. This is different from the case of an NLO color singlet production, which are covered in Ref. [11]. There, all NLP terms (also those that are beyond NLP LL order) are captured. As is well known, in the present case, the possibility of having final state colinear emissions creates an NLP term that is of NLL order. This is a simple consequence of the fact that, in processes with final state unobserved emissions, the threshold expansion does not coincide with the soft expansion of the matrix element. As our formalism only accounts for emissions that are manifestly soft or next-to-soft, we do not capture these NLL terms. To complete the set of NLL contributions, one needs to take into account hard-collinear information via the addition of a gluon jet function.

Upon inspection of the other LP channel, we find that the soft quark amplitude of Eq. (1.1) is needed to account for all NLP terms in the NLO expression. There, we also see that there is a soft quark interference contribution, due to the presence of more than two colored partons in the hard scattering. By combining the soft gluon and quark amplitudes, we can correctly account for the NLP contributions of all 7 partonic subchannels of the prompt photon process. In addition, we have applied the formalism to deep-inelastic scattering, and $e^{+} e^{-} \rightarrow q \bar{q}$, and recover all LL NLP terms in those processes as well.

\section{Numerical impact of NLP terms to the jointly resummed production of prompt photons}

We consider the inclusive transverse momentum distribution of photons produced at fixed $p_{T}$ in proton-proton collisions

$$
h_{A}\left(p_{A}\right)+h_{B}\left(p_{B}\right) \rightarrow \gamma\left(p_{\gamma}\right)+X
$$

where $h_{A, B}$ refers to the two incoming protons and $X$ to the unobserved part of the final state. The partonic momenta $p_{a}$ and $p_{b}$ are related to the hadronic ones via $p_{a}=x_{a} p_{A}$ and $p_{b}=x_{b} p_{B}$. In the center of mass frame of the initial state particles it is convenient to parameterize the photon momentum $p_{\gamma}$ as

$$
p_{\gamma}=\left(p_{T} \cosh (\eta), \mathbf{p}_{T}, p_{T} \sinh (\eta)\right)
$$

where $\mathbf{p}_{T}$ and $\eta$ are the transverse momentum and pseudorapidity of the photon, and we denote $\left|\mathbf{p}_{T}\right|$ by $p_{T}$. The minimum value of $\cosh (\eta)$ is equal to 1 , therefore the partonic threshold is at $s=4 p_{T}^{2}$. The distance $\xi$ to threshold in Eq. (1) can be written as $\xi=1-\hat{x}_{T}^{2}$, where $\hat{x}_{T}=\frac{2 p_{T}}{\sqrt{s}}$. The hadronic equivalent of $\hat{x}_{T}$ is denoted as $x_{T}=\frac{2 p_{T}}{\sqrt{S}}$. The photon can be created directly (see Fig. 1), or in a fragmentation process in $2 \rightarrow 2$ parton scattering. The combined differential cross section for prompt photon production is therefore a sum of two parts

$$
p_{T}^{3} \frac{\mathrm{d} \sigma_{A B \rightarrow \gamma+X}^{(\text {comb })}}{\mathrm{d} p_{T}}=p_{T}^{3} \frac{\mathrm{d} \sigma_{A B \rightarrow \gamma+X}^{(\text {direct })}}{\mathrm{d} p_{T}}+p_{T}^{3} \frac{\mathrm{d} \sigma_{A B \rightarrow \gamma+X}^{(\text {frag })}}{\mathrm{d} p_{T}} .
$$

Joint resummation takes into account the recoil of the hard scattering process against additional radiated partons with collective transverse momentum $\mathbf{Q}_{T}$. This implies that the photon transverse momentum to be produced by the hard scattering is only $\mathbf{p}_{T}^{\prime}=\mathbf{p}_{T}-\mathbf{Q}_{T} / 2$, which effectively lowers 
the partonic threshold. The corresponding new scaling variable is $\tilde{x}_{T}^{2}=4 p_{T}^{\prime 2} / Q^{2}$, where $Q$ is the invariant mass of the photon-parton pair in the recoil frame. The variables $x_{T}^{2}$ and $\tilde{x}_{T}^{2}$ are related by

$$
\tilde{x}_{T}^{2}=x_{T}^{2}\left(\frac{S}{Q^{2}} \frac{p_{T}^{\prime 2}}{p_{T}^{2}}\right) .
$$

It was shown in $[38,39]$ that resummation of threshold and recoil logarithms can be jointly performed for sufficiently small values of $\left|\mathbf{Q}_{T}\right| \equiv Q_{T}$. The expression for the joint-resummed $p_{T}$ distribution of the direct component reads $[39,40]$

$$
\begin{aligned}
& p_{T}^{3} \frac{\mathrm{d} \sigma_{A B \rightarrow \gamma+X}^{\text {(direct,joint) }}\left(x_{T}^{2}\right)}{\mathrm{d} p_{T}}=\frac{p_{T}^{4}}{8 \pi S^{2}} \sum_{a, b} \int_{\mathscr{C}} \frac{\mathrm{d} N}{2 \pi i} f_{a / A}\left(N, \mu_{F}\right) f_{b / B}\left(N, \mu_{F}\right) \\
& \quad \times \int \frac{\mathrm{d}^{2} \mathbf{Q}_{T}}{(2 \pi)^{2}}\left(\frac{S}{4\left|\mathbf{p}_{T}-\mathbf{Q}_{T} / 2\right|^{2}}\right)^{N+1} \int_{0}^{1} \mathrm{~d} \tilde{x}_{T}^{2}\left(\tilde{x}_{T}^{2}\right)^{N} \frac{\left|\mathscr{M}_{a b \rightarrow \gamma d}\left(\tilde{x}_{T}^{2}\right)\right|^{2}}{\sqrt{1-\tilde{x}_{T}^{2}}} C_{\delta}^{(a b \rightarrow \gamma d)}\left(\alpha_{s}, \tilde{x}_{T}^{2}\right) \\
& \quad \times \int \mathrm{d}^{2} \mathbf{b} \mathrm{e}^{i \mathbf{b} \cdot \mathbf{Q}_{T}} \boldsymbol{\theta}\left(\bar{\mu}-\left|\mathbf{Q}_{T}\right|\right) P_{a b d}\left(N, b, Q, \mu_{F}, \mu\right)
\end{aligned}
$$

where each of the components are defined in Ref. [41]. The logarithmic contributions are captured by the function $P_{a b d}$. The expression for the fragmentation contribution is similar to Eq. (3.4), with the addition that one needs to include a fragmentation function and take into account multiple color structures.

The singularity that is found present at $p_{T}=Q_{T} / 2$ is treated by approximating the kinematic factor [42]

$$
\begin{aligned}
\left(\frac{S}{4\left(\mathbf{p}_{T}-\mathbf{Q}_{T} / 2\right)^{2}}\right)^{N+1} & =\left(\frac{4 p_{T}^{2}}{S}\right)^{-N-1}\left(1-\frac{\mathbf{p}_{T} \cdot \mathbf{Q}_{T}}{p_{T}^{2}}+\frac{Q_{T}^{2}}{4 p_{T}^{2}}\right)^{-N-1} \\
& \simeq\left(x_{T}^{2}\right)^{-N-1} \exp \left[(N+1) \frac{\mathbf{p}_{T} \cdot \mathbf{Q}_{T}}{p_{T}^{2}}\left[1+\mathscr{O}\left(Q_{T} / p_{T}\right)\right]\right]
\end{aligned}
$$

Using Eq. (3.5) in Eq. (3.4), one sees that the integral over $\mathrm{d}^{2} \mathbf{Q}_{T}$ produces the delta function $\delta\left(\mathbf{b}-i(N+1) \mathbf{p}_{T} / p_{T}^{2}\right)$. This delta function may be used to perform the integral over $\mathrm{d}^{2} \mathbf{b}$, which fixes $b=i(N+1) / p_{T}$.

The initial state exponent in $P_{a b d}$ may then be treated as follows

$$
\begin{aligned}
E_{a}^{\mathrm{PT}}\left(N, b, Q, \mu_{F}, \mu\right)= & \int_{0}^{Q^{2}} \frac{\mathrm{d} k_{T}^{2}}{k_{T}^{2}} A_{a}\left(\alpha_{s}\left(k_{T}^{2}\right)\right)\left[J_{0}\left(b k_{T}\right) K_{0}\left(\frac{2 N k_{T}}{Q}\right)+\ln \left(\frac{\bar{N} k_{T}}{Q}\right)\right] \\
& -\ln \bar{N} \int_{\mu_{F}^{2}}^{Q^{2}} \frac{\mathrm{d} k_{T}^{2}}{k_{T}^{2}} A_{a}\left(\alpha_{s}\left(k_{T}^{2}\right)\right) \\
\equiv & E_{a}^{\mathrm{joint}}(N, b, Q, \mu)+E_{a}^{\mathrm{DGLAP}}\left(N, Q, \mu_{F}, \mu\right) .
\end{aligned}
$$

The exponent $E_{a}^{\text {joint }}$ with $b=i(N+1) / p_{T}$ reads

$$
E_{a}^{\mathrm{joint}}\left(N, b=i \frac{N+1}{p_{T}}, Q, \mu\right)=\int_{0}^{Q^{2}} \frac{\mathrm{d} k_{T}^{2}}{k_{T}^{2}} A_{a}\left(\alpha_{S}\left(k_{T}^{2}\right)\right)\left[K_{0}\left(\frac{2 N k_{T}}{Q}\right)+\ln \left(\frac{\bar{N} k_{T}}{Q}\right)\right]
$$




$$
\begin{gathered}
+\int_{0}^{Q^{2}} \frac{\mathrm{d} k_{T}^{2}}{k_{T}^{2}} A_{a}\left(\alpha_{s}\left(k_{T}^{2}\right)\right)\left[I_{0}\left(\frac{(N+1) k_{T}}{p_{T}}\right)-1\right] K_{0}\left(\frac{N k_{T}}{p_{T}}\right) . \\
\equiv E_{a}^{\text {leading }}(N, Q, \mu)+E_{a}^{\text {recoil }}(N, Q, \mu) .
\end{gathered}
$$

The term $E^{\text {recoil }}$ contributes at NLP LL in Mellin space, which is simply a consequence of the large $N$-expansion of the integrals that need to be performed. The term $E_{a}^{\text {leading }}$, when combined with $E_{a}^{\text {DGLAP }}$ in Eq. (3.6), can be recognized as the exponent for threshold resummation for the initial state

$$
\begin{aligned}
E_{a}^{\text {thres }}\left(N, Q, \mu_{F}, \mu\right) & =-\int_{1 / \bar{N}}^{1} \frac{\mathrm{d} y}{y} \int_{\mu_{F}^{2}}^{y^{2} Q^{2}} \frac{\mathrm{d} k_{T}^{2}}{k_{T}^{2}} A_{a}\left(\alpha_{S}\left(k_{T}^{2}\right)\right) \\
& =\int_{0}^{1} \mathrm{~d} z \frac{z^{N-1}-1}{1-z} \int_{\mu_{F}^{2}}^{(1-z)^{2} Q^{2}} \frac{\mathrm{d} k_{T}^{2}}{k_{T}^{2}} A_{a}\left(\alpha_{s}\left(k_{T}^{2}\right)\right) .
\end{aligned}
$$

We may then modify $E_{a}^{\text {thres }}$ (and similarly, the exponent that is used for logarithmic contributions of final state radiation) to include NLP terms. The modifications we propose for the initial state are described in detail in Ref. [41], and summarized by:

1) A modified initial state resummation coefficient where $\frac{z^{N-1}-1}{1-z} \rightarrow \frac{z^{N-1}-1}{1-z}-z^{N-1}$.

2a) An extended evolution of the initial state partons from $\mu_{F}$ to $Q / \bar{N}$, keeping only the diagonal terms in the splitting functions. This method should give the same result as item 1 for $\mu_{F}=Q$.

2b) Evolve the partons from $\mu_{F}$ to $Q / \bar{N}$, including the non-diagonal terms in the singlet case. The splitting functions are expanded to $\mathscr{O}(1 / N)$.

2c) Evolve the partons from $\mu_{F}$ to $Q / \bar{N}$ and keep the full form of all splitting functions.

The study of the prompt photon process at NLO shows that these modifications do not capture all LL NLP terms. The LL NLP terms that we do not catch can be classified in three categories. The LL NLP terms that we do not catch can be classified in three categories. First, some of the terms have a non-collinear origin. These cannot be reproduced via a modification of the splitting/fragmentation functions, as these only contain collinear effects. A second category follows from the modification of the hard scattering kinematics as a result of the next-to-soft gluon emission. The $\mathscr{O}(1-z)$ expansion of the Born function is multiplied with a LL LP term, which results in a class of NLP logarithms. Another source of LL NLP terms in Mellin space that is not included is due to NLP phase space effects. These originate from the approximation of the soft function by transforming it into Mellin space and only taking the first order as $N \rightarrow \infty$. The Mellin moment is taken with respect to $x_{T}^{2}=4 p_{T}^{2} / s$, whose connection to the $v$ and $w$ parameterization of Ref. [43] reads

$$
\tilde{\sigma}(N)=\int_{0}^{1} \mathrm{~d} x_{T}^{2}\left(x_{T}^{2}\right)^{N-1} \frac{p_{T}^{3} \mathrm{~d} \sigma\left(p_{T}\right)}{\mathrm{d} p_{T}}=\frac{1}{2} \int_{0}^{1} \mathrm{~d} v \int_{0}^{1} \mathrm{~d} w(4 v(1-v) w)^{N+1} \frac{s \mathrm{~d} \sigma(v, w)}{\mathrm{d} v \mathrm{~d} w} .
$$

The wide-angle soft coefficient $g_{(a b c)}^{(1)}$ has a dependence on $v$ and $w$ that reads [39]

$$
\begin{aligned}
& g_{q \bar{q} g}^{(1)}(v, w)=\frac{\alpha_{s}}{\pi} \ln (v w(1-v)) \\
& g_{q g q}^{(1)}(v, w)=\frac{\alpha_{s}}{\pi}\left[2 C_{F} \ln (v w)+C_{A} \ln \left(\frac{1-v}{v w}\right)\right] .
\end{aligned}
$$


The Mellin transform of these functions involve

$$
\int_{0}^{1} \mathrm{~d} v(4 v(1-v))^{N+1} f(v)=f\left(\frac{1}{2}\right)+\mathscr{O}\left(\frac{1}{N}\right) .
$$

Therefore, at LP, $v$ may be fixed to $1 / 2$, which is done to obtain the wide-angle soft function. Corrections to this start at $\mathscr{O}(1 / N)$ terms and appear for $v \neq 1 / 2$. Such $\mathscr{O}(1 / N)$ contributions originate in $(v, w)$-space from an NLL LP contribution, but in Mellin space it resembles an LL NLP contribution. To see this, note that the Mellin transform of the plus distribution is of NLL LP:

$$
\int_{0}^{1} \mathrm{~d} w w^{N} \frac{1}{(1-w)_{+}} \simeq-\ln \bar{N}+\mathscr{O}\left(\frac{1}{N}\right) .
$$

However, the $\mathscr{O}(1 / N)$ term created by the Mellin transform in Eq. (3.12) multiplied with NLL LP contribution creates a term that is of LL NLP in Mellin space. We do not take into account this contribution at present. However, we do capture all LL NLP effects that have a collinear origin for both gluon and quark emission.

The foregoing shows that the connection between (N)LP contributions in momentum space and those in Mellin space deserves further attention: LL NLP contributions in Mellin space arise from NLL LP contributions in momentum space. Moreover, the recoil correction, which is of NLL in momentum space, creates an LP NNLL + NLP LL contribution in Mellin space.

For the numerical results we consider the case of the LHC operating at a center of mass energy of $\sqrt{S}=13 \mathrm{TeV}$. For the parton distributions we use the central MMHT set [44], corresponding to $\alpha_{s}\left(M_{Z}^{2}\right)=0.120$. For the fragmentation function we use the results of [45]. We set the factorization scale $\mu_{F}$ equal to the renormalization scale $\mu$. We checked that the results presented below do not change significantly when $\mu_{F} \neq \mu$. The hard scale $Q$ is set to $2 p_{T}$ and $\mu$ is chosen equal to $Q$ unless stated otherwise.

The result after the inclusion of both the initial and final state NLP terms can be seen in Fig. 2, where we show the combined joint-resummed $p_{T}$ distribution for three scales. We observe that the ratio of option 1 to the LP NLL resummed result amounts to a $10-20 \%$ correction and is robust under scale variations. On the other hand, the ratios that are obtained with the evolution approaches (option $2 \mathrm{~b}$ and $2 \mathrm{c}$ ) are highly scale dependent. In Fig. 3 we can observe that the scale dependence of these ratios is in fact caused by the LP NLL joint-resummed $p_{T}$ distribution. Remarkably, the distributions that are obtained with option $2 b$ (or $2 c$ ) are very robust under variations of scale. It is interesting to observe a significant decrease in scale dependence only after including the offdiagonal components of the splitting functions, which deserves further study.

\section{Conclusion}

We see that the LL contributions at LP and NLP are correctly described at NLO by the NLP amplitude (Eq. (1.1)). The general NLO cross section for soft gluon emissions is given in terms of the LO amplitude with shifted momenta, similar as to what was found for color singlet production processes in Ref. [11]. When soft quark emissions are present, we need to complement the nextto-soft gluon amplitude with soft quark amplitudes, which also give rise to LL NLP contributions. 

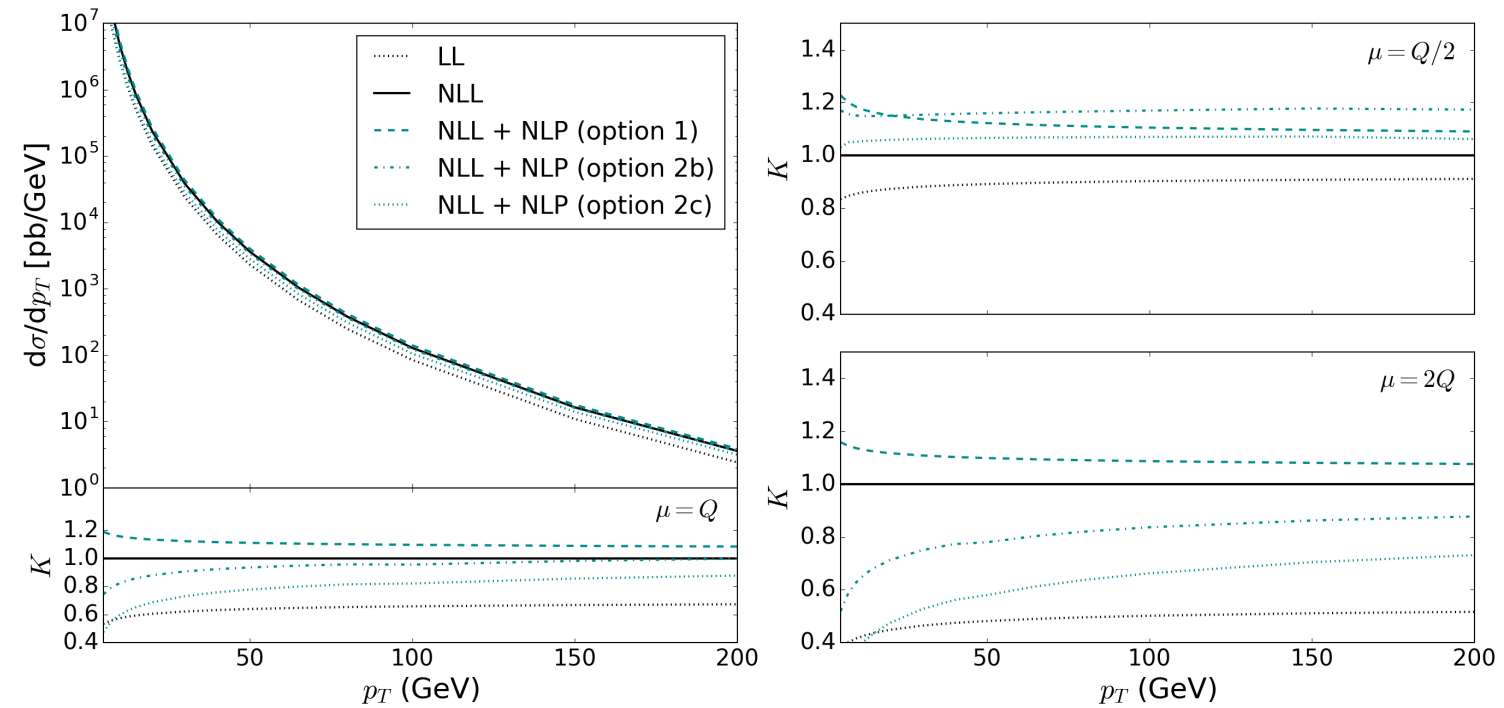

Figure 2: Combined joint-resummed $p_{T}$ distribution for LP resummation (black) and inclusion of NLP effects for both the initial and final state (blue). The bottom pannel and the plots on the right show the ratio $K$ with respect to the LP NLL resummed result for three choices of scale: $\mu=Q$ (left), $\mu=Q / 2$ (top right) and $\mu=2 Q$ (lower right).
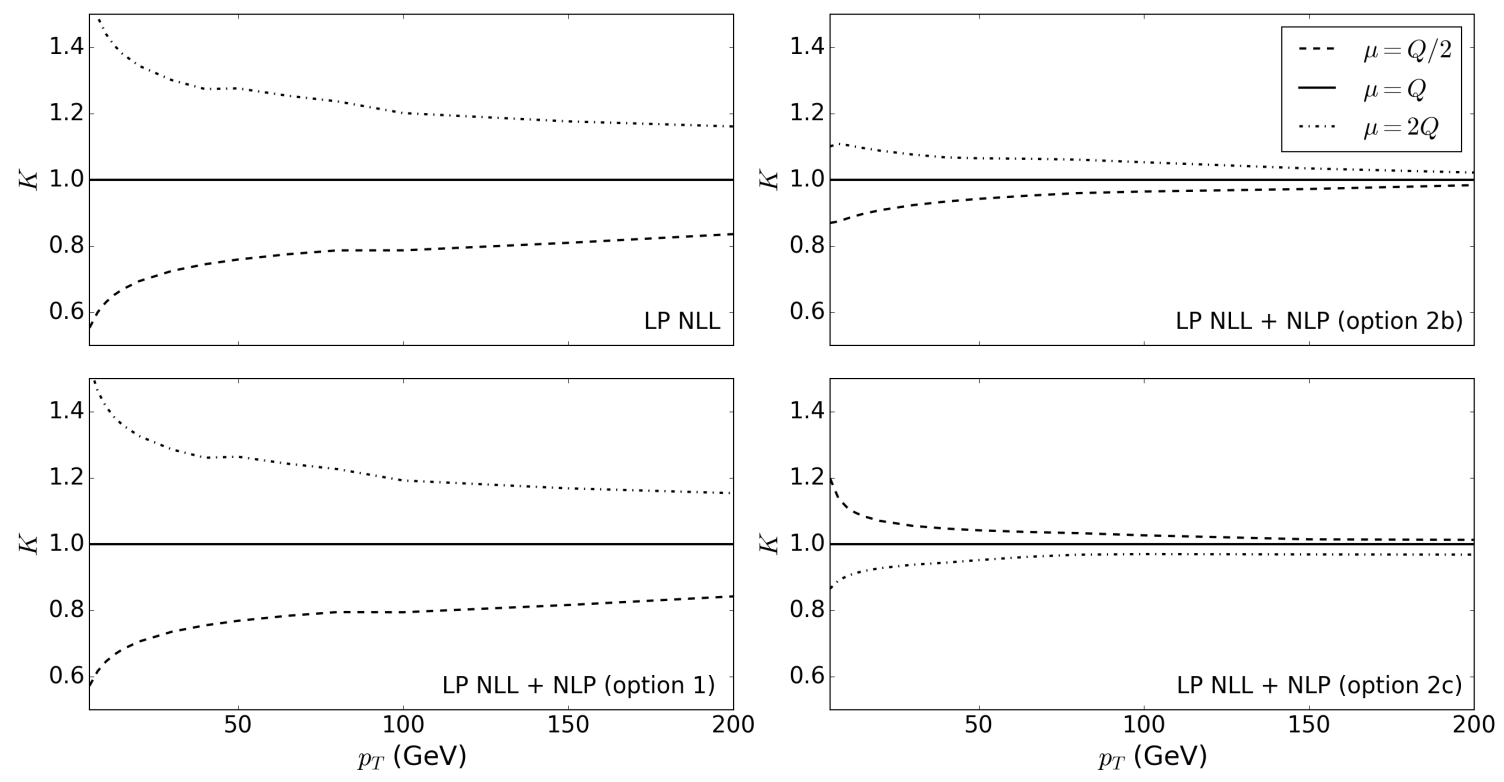

Figure 3: The ratio $K$ of the combined joint-resummed $p_{T}$ distribution for $\mu=Q / 2$ (dashed) and $\mu=2 Q$ (dash-dotted) to $\mu=Q$ (solid). Four levels of accuracy are shown: LP NLL (top left), option 1 (lower left), option $2 \mathrm{~b}$ (top right) and option $2 \mathrm{c}$ (lower left). 
To assess the numerical impact of NLP contributions, we have included NLP contributions that have a (next-to-)soft-collinear origin to the jointly resummed prompt photon production process. The correction obtained by including (next-to-)soft collinear gluon emissions is about $10 \%$ for high $p_{T}$ values and $20 \%$ for low $p_{T}$. The correction that is obtained by including both gluon and quark emission at NLP LL accuracy diminishes at central scale, which is caused by a sign difference between the diagonal and off-diagonal part of the splitting functions. However, at different scale choices, the correction can grow to more than $40 \%$ for small $p_{T}$ values and $20 \%$ for large $p_{T}$. This is due to the fact that the LP NLL resummed result is heavily scale dependent, and this dependence is greatly reduced by the inclusion of soft quark emission.

\section{References}

[1] Yu. L. Dokshitzer, G. Marchesini, and G. P. Salam, Revisiting parton evolution and the large-x limit, Phys. Lett. B634 (2006) 504-507, [hep-ph/ 0511302$].$

[2] E. Laenen, L. Magnea, and G. Stavenga, On next-to-eikonal corrections to threshold resummation for the Drell-Yan and DIS cross sections, Phys. Lett. B669 (2008) 173-179, [arXiv: 0807.4412 ].

[3] E. Laenen, G. Stavenga, and C. D. White, Path integral approach to eikonal and next-to-eikonal exponentiation, JHEP 0903 (2009) 054, [arXiv : 0811 . 2067].

[4] E. Laenen, L. Magnea, G. Stavenga, and C. D. White, Next-to-eikonal corrections to soft gluon radiation: a diagrammatic approach, JHEP 1101 (2011) 141, [arXiv:1010.1860].

[5] D. Bonocore, E. Laenen, L. Magnea, L. Vernazza, and C. D. White, The method of regions and next-to-soft corrections in Drell-Yan production, Phys. Lett. B742 (2015) 375-382, [arXiv:1410.6406].

[6] A. J. Larkoski, D. Neill, and I. W. Stewart, Soft theorems from effective field theory, JHEP 1506 (2015) 077, [arXiv:1412.3108].

[7] D. Bonocore, E. Laenen, L. Magnea, S. Melville, L. Vernazza, and C. D. White, A factorization approach to next-to-leading-power threshold logarithms, JHEP 06 (2015) 008, [arXiv:1503.05156].

[8] D. Bonocore, E. Laenen, L. Magnea, L. Vernazza, and C. D. White, Non-abelian factorisation for next-to-leading-power threshold logarithms, JHEP 12 (2016) 121, [arXiv: 1610.06842 ].

[9] I. Moult, L. Rothen, I. W. Stewart, F. J. Tackmann, and H. X. Zhu, Subleading power corrections for N-jettiness subtractions, Phys. Rev. D95 (2017), no. 7 074023, [arXiv: 1612.00450 ].

[10] R. Boughezal, X. Liu, and F. Petriello, Power corrections in the N-jettiness subtraction scheme, JHEP 1703 (2017) 160, [arXiv:1612.02911].

[11] V. Del Duca, E. Laenen, L. Magnea, L. Vernazza, and C. D. White, Universality of next-to-leading power threshold effects for colourless final states in hadronic collisions, JHEP 11 (2017) 057, [arXiv:1706.04018].

[12] I. Moult, L. Rothen, I. W. Stewart, F. J. Tackmann, and H. X. Zhu, N-jettiness subtractions for $g g \rightarrow H$ at subleading power, Phys. Rev. D97 (2018), no. 1 014013, [arXiv: 1710.03227 ].

[13] M. Beneke, M. Garny, R. Szafron, and J. Wang, Anomalous dimension of subleading-power N-jet operators, JHEP 03 (2018) 001, [arXiv: 1712.04416 ]. 
[14] I. Feige, D. W. Kolodrubetz, I. Moult, and I. W. Stewart, A complete basis of helicity operators for subleading factorization, JHEP 1711 (2017) 142, [arXiv: 1703.03411 ].

[15] I. Moult, I. W. Stewart, and G. Vita, A subleading operator basis and matching for gg $\rightarrow H, J H E P$ 1707 (2017) 067, [arXiv:1703.03408].

[16] C.-H. Chang, I. W. Stewart, and G. Vita, A subleading power operator basis for the scalar quark current, JHEP 1804 (2018) 041, [arXiv: 1712 .04343].

[17] M. Beneke, M. Garny, R. Szafron, and J. Wang, Anomalous dimension of subleading-power $N$-jet operators. Part II, JHEP 11 (2018) 112, [arXiv: 1808 . 04742 ].

[18] I. Moult, I. W. Stewart, G. Vita, and H. X. Zhu, First Subleading Power Resummation for Event Shapes, JHEP 08 (2018) 013, [arXiv: 1804.04665$].$

[19] M. A. Ebert, I. Moult, I. W. Stewart, F. J. Tackmann, G. Vita, and H. X. Zhu, Power Corrections for $N$-Jettiness Subtractions at $\mathscr{O}\left(\alpha_{s}\right)$, JHEP 12 (2018) 084, [arXiv: 1807.10764 ].

[20] M. A. Ebert, I. Moult, I. W. Stewart, F. J. Tackmann, G. Vita, and H. X. Zhu, Subleading power rapidity divergences and power corrections for $q_{T}$, JHEP 04 (2019) 123, [arXiv: 1812.08189 ].

[21] A. Bhattacharya, I. Moult, I. W. Stewart, and G. Vita, Helicity Methods for High Multiplicity Subleading Soft and Collinear Limits, arXiv:1812.06950.

[22] R. Boughezal, A. Isgrò, and F. Petriello, Next-to-leading-logarithmic power corrections for N-jettiness subtraction in color-singlet production, Phys. Rev. D97 (2018), no. 7076006 , [arXiv:1802.00456].

[23] M. Beneke, M. Garny, R. Szafron, and J. Wang, Violation of the Kluberg-Stern-Zuber theorem in SCET, JHEP 09 (2019) 101, [arXiv: 1907 . 05463].

[24] R. Boughezal, A. Isgrò, and F. Petriello, Next-to-leading power corrections to $V+1$ jet production in $N$-jettiness subtraction, arXiv:1907.12213.

[25] I. Moult, I. W. Stewart, and G. Vita, Subleading Power Factorization with Radiative Functions, arXiv:1905.07411.

[26] L. Cieri, C. Oleari, and M. Rocco, Higher-order power corrections in a transverse-momentum cut for colour-singlet production at NLO, Eur. Phys. J. C79 (2019), no. 10 852, [arXiv: 1906.09044 ].

[27] I. Moult, I. W. Stewart, G. Vita, and H. X. Zhu, The Soft Quark Sudakov, arXiv: 1910.14038.

[28] M. Beneke, A. Broggio, S. Jaskiewicz, and L. Vernazza, Threshold factorization of the Drell-Yan process at next-to-leading power, arXiv:1912.01585.

[29] I. Moult, G. Vita, and K. Yan, Subleading Power Resummation of Rapidity Logarithms: The Energy-Energy Correlator in $\mathscr{N}=4$ SYM, arXiv:1912.02188.

[30] M. Beneke, A. Broggio, M. Garny, S. Jaskiewicz, R. Szafron, L. Vernazza, and J. Wang, Leading-logarithmic threshold resummation of the Drell-Yan process at next-to-leading power, JHEP 1903 (2019) 043, [arXiv:1809.10631].

[31] M. Beneke, M. Garny, S. Jaskiewicz, R. Szafron, L. Vernazza, and J. Wang, Leading-logarithmic threshold resummation of Higgs production in gluon fusion at next-to-leading power, arXiv:1910.12685.

[32] N. Bahjat-Abbas, D. Bonocore, J. Sinninghe Damsté, E. Laenen, L. Magnea, L. Vernazza, and C. D. White, Diagrammatic resummation of leading-logarithmic threshold effects at next-to-leading power, arXiv:1905.13710. 
[33] M. van Beekveld, W. Beenakker, E. Laenen, and C. D. White, Next-to-leading power threshold effects for inclusive and exclusive processes with final state jets, arXiv: 1905.08741.

[34] S. Weinberg, Infrared photons and gravitons, Phys. Rev. 140 (1965) B516-B524.

[35] E. Casali, Soft sub-leading divergences in Yang-Mills amplitudes, JHEP 1408 (2014) 077, [arXiv:1404.5551].

[36] F. Cachazo and A. Strominger, Evidence for a new soft graviton theorem, arXiv: 1404.4091.

[37] C. D. White, Factorization properties of soft graviton amplitudes, JHEP 1105 (2011) 060, [arXiv:1103.2981].

[38] E. Laenen, G. Sterman, and W. Vogelsang, Higher-order qcd corrections in prompt photon production, Phys. Rev. Lett. 84 (2000) 4296, [hep-ph / 0002078 ].

[39] E. Laenen, G. Sterman, and W. Vogelsang, Recoil and threshold corrections in short-distance cross sections, Phys. Rev. D63 (2001) 114018, [http: / / arXiv.org/abs/hep-ph/ 010080 ].

[40] R. Basu, E. Laenen, A. Misra, and P. Motylinski, Soft-collinear effects in prompt photon production, Phys. Rev. D76 (2007) 014010, [arXiv: 0704 . 3180].

[41] M. van Beekveld, W. Beenakker, R. Basu, E. Laenen, A. Misra, and P. Motylinski, Next-to-leading power threshold effects for resummed prompt photon production, arXiv: 1905.11771.

[42] G. Sterman and W. Vogelsang, Recoil and power corrections in high-x(t) direct-photon production, Phys. Rev. D71 (2005) 014013, [hep-ph/ 0409234 ].

[43] L. E. Gordon and W. Vogelsang, Polarized and unpolarized prompt photon production beyond the leading order, Phys. Rev. D48 (1993) 3136-3159.

[44] L. A. Harland-Lang, A. D. Martin, P. Motylinski, and R. S. Thorne, Parton distributions in the LHC era: MMHT 2014 PDFs, Eur. Phys. J. C75 (2015), no. 5 204, [arXiv: 1412.3989 ].

[45] M. Gluck, E. Reya, and A. Vogt, Parton fragmentation into photons beyond the leading order, Phys. Rev. D48 (1993) 116-128. 